\title{
Editorial: Advances in the statistical modelling of spatial interaction data
}

\author{
Roberto Patuelli • Giuseppe Arbia
}

Received: 11 March 2013/Accepted: 21 March 2013/Published online: 17 April 2013

(C) Springer-Verlag Berlin Heidelberg 2013

The papers appearing in this special issue of the Journal of Geographical Systems come from an International Exploratory Workshop entitled 'Advances in the Statistical Modelling of Spatial Interaction Data', organized at the University of Lugano (Switzerland) on 15-16 September 2011. The aim of the workshop was to provide a common ground for discussion between researchers active in this field and to discuss some of the most recent developments. As guest editors, we would particularly like to thank the authors for contributing to the scientific success and cordial climate of the workshop and for accepting to be included in this special issue. We also wish to thank the referees for their work and for their important contribution to what we believe represents an interesting selection of papers touching several frontier topics in the field.

Spatial interaction (SI) models have been, for many decades, the workhorse of regional science in the analysis of the interactions observed in space between social and economic agents. As a matter of fact, SI is at the basis of all processes involving choices in regional science problems, and it may equally refer to all economic agents (firms, workers or households) and to their behaviour in various fields such as commuting, shopping trips, journey-to-work, migration, housing choices and international and interregional trade.

\footnotetext{
R. Patuelli $(\bowtie)$

Department of Economics, University of Bologna, Rimini Campus,

via Angherà 22, Rimini RN, Italy

e-mail: roberto.patuelli@unibo.it

R. Patuelli

The Rimini Centre for Economic Analysis (RCEA), Rimini, Italy

G. Arbia

Department of Statistics, Università Cattolica del Sacro Cuore, Rome, Italy

e-mail: giuseppe.arbia@rm.unicatt.it
} 
While originating already at the end of the 19th century along with the Newtonian paradigm, SI models have only acquired theoretical foundations in the second half of the 20th century with the contributions of Stewart (1941), Isard (1960) and Wilson $(1967,1970)$, thus receiving sound economic motivations based on probabilistic, discrete choice and entropy maximization concepts. For a coherent state-of-the-art overview of the field that has witnessed the progression from gravity models to entropy and random utility maximizing models and finally to models based on neurocomputing principles, see Fischer and Reggiani (2004).

Origin-destination flows are not independent, because flows are fundamentally spatial in nature. Spatial dependence in flows relates to correlations among flows between locations that are neighbouring a given origin-destination pair. Thus, a failure to account for spatial dependence in model specifications may lead to biased parameter estimates and incorrect conclusions. One way to address this problem is to incorporate spatial dependence by specifying a spatial process that governs the spatial interaction variable. Another way to dealing with spatial dependence in origin-destination flows is to specify a spatial process for the disturbance terms, according to a spatial autoregressive structure. A third way to address spatial dependence in origin-destination flows involves eigenvector spatial filtering (for details on different ways to deal with the problem, see LeSage and Fischer 2009).

This special issue includes six contributions. The first two articles focus on statistical aspects related to SI analysis. The article by Bavaud presents a new approach to spatial autocorrelation testing for weighted networks, which are specified by a symmetric, normalized exchange matrix. This approach consists of expressing Moran's I in terms of spatial modes, defined as the projection of the variable on the eigenvectors of the exchange matrix, as illustrated on Swiss migratory flows and on blood group A data for Ireland. In the second article, Arbia and Petrarca introduce for the first time a rigorous analysis of the effects of the modifiable areal unit problem (MAUP) in SI models. In particular, they focus on one dimension of the MAUP, the so-called scale problem, which pertains to the different results of statistical analyses at different levels of geographical aggregation. Through a theoretical analysis illustrated with a set of simulated data, they model the increase in the mean flows of interaction observed when aggregating the data at different scales. They observe that such an increase is maximized in the presence of negative spatial autocorrelation in the origin and destination variables.

The two subsequent articles provide new views on the estimation of SI models. LeSage and Llano develop an SI model that makes use of origin and destination spatially structured latent factors. They estimate the SI model within a Bayesian hierarchical framework, by imposing a spatial autoregressive structure for the random effects incorporating the latent effects. Griffith and Fischer shift attention to singly and doubly constrained exponential SI model variants for situations involving flows taking the form of counts. The paper establishes theoretical connections between the constrained SI model versions with balancing factors, fixed effects represented by binary location-specific indicator variables and random effects. The authors illustrate these connections with an empirical example, while accounting for spatial dependence among flows using eigenvector spatial filtering. 
The final two articles in the special issue present advanced applications of spatial econometrics within SI models. De La Mata and Llano study interregional trade flows of services in Spain and investigate whether social networks (measured via internal migration flows) influence such flows as seen in international trade. They show that applying spatial econometric techniques using information on past migration flows helps account for significant spatial dependence in the data. Moreover, they find that the deterrence effect of distance is diminished when accounting for the stock of migrants in the origins and destinations. In the last article, Patuelli, Mussoni and Candela present an analysis of interregional tourism flows in Italy, focussing on the effect of sites included in UNESCO's World Heritage List. By modelling intervening opportunities in the tourists' evaluation of listed sites, they show that spatial competition between regions on the basis of their certified cultural endowment dominates over spatial complementarity and that such competition can overcome the direct positive effects of site listing, depending on the type and geographical extent of the assumed SI process.

Finally, we are grateful to Rico Maggi and the University of Lugano for supporting our initiative and to the SNSF for generously funding it. We are confident that the contributions provided here will represent a useful reference point for those who are interested in exploring spatial dependence issues in SI data or are looking for guidance on the choice of a suitable econometric specification for practical purposes.

\section{References}

Fischer MM, Reggiani A (2004) Spatial interaction models: from the gravity to the neural network approach. In: Capello R, Nijkamp P (eds) Urban dynamics and growth: advances in urban economics. Elsevier, Amsterdam Oxford, pp 319-346

Isard W (1960) Methods of regional analysis. MIT Press, Cambridge

LeSage JP, Fischer MM (2009) Spatial econometric methods for modeling of origin-destination flows. In: Fischer MM, Getis A (eds) Handbook of applied spatial analysis: software tools, methods and applications. Springer, Heidelberg,Dordrecht, London, New York, pp 409-433

Stewart JQ (1941) On inverse distance variation for certain social influences. Science 93(2404):89-90

Wilson AG (1967) A statistical theory of spatial distribution models. Transp Res 1(3):253-269

Wilson AG (1970) Entropy in urban and regional modelling. Pion, London 